\title{
NILPOTENT ACTION BY AN AMENABLE GROUP AND EULER CHARACTERISTIC
}

\author{
by JONG BUM LEE*
}

(Received 10th January 1997)

We prove two types of vanishing results for the Euler characteristic.

1991 Mathematics subject classification: 57 S25.

\section{Introduction}

Let $X$ be a finite connected simplicial complex, $\Gamma=\pi_{1}(X)$ its fundamental group, $\tilde{X}$ its universal covering space. Then $\Gamma$ acts freely on $\tilde{X}$ as simplicial automorphisms and on the cohomology group $H^{*}(\tilde{X})$. In this note we establish the following vanishing results for the Euler characteristic $\chi(X)$ of $X$.

Theorem 1.1. If $\Gamma=\pi_{1}(X)$ is an amenable group and $\Gamma$ contains an infinite normal subgroup $A$ which acts nilpotently on $H^{*}(\tilde{X})$, then the reduced $\ell_{2}$-cohomology spaces $\bar{H}_{(2)}^{*}(\tilde{X}: \Gamma)$ are trivial. In particular, the Euler characteristic $\chi(X)$ of $X$ vanishes.

Theorem 1.2. If $\Gamma=\pi_{1}(X)$ acts nilpotently on $H^{*}(\tilde{X})$ and contains a normal subgroup $A$ such that the quotient group $\Gamma / A$ is infinite amenable and $A$ is $\Gamma$-nilpotent, then the Euler characteristic $\chi(X)$ of $X$ vanishes.

A discrete group $G$ is called amenable if it admits a left invariant mean for $\ell_{\infty}(G)$, i.e., if there exists a functional $m: \ell_{\infty}(G) \rightarrow \mathbb{R}$ satisfying $m\left(\chi_{G}\right)=1$ and $m(\phi x)=m(\phi)$ for all $x \in G$ and $\phi \in \ell_{\infty}(G)$. For example finite, Abelian, and solvable groups are amenable groups. A group containing a non-Abelian free subgroup is not amenable. A left invariant mean for a finite group $G$ is obtained by letting $m(\phi)=\frac{1}{|G|} \sum_{x \in G} \phi(x)$. For further details on amenable groups we refer to [9].

If $\Gamma$ is infinite amenable and $X$ is aspherical, then Cheeger and Gromov [2] and Eckmann [5] showed that $\chi(X)=0$. If $\Gamma$ contains a nontrivial torsion-free Abelian normal subgroup which acts nilpotently on $H^{*}(\tilde{X})$, then Eckmann [4] showed that

* Supported in part by Sogang University Research Grant, by BSRI-96-1434, KOREA, and by TGRCKOSEF, KOREA. 
$\chi(X)=0$. If $\Gamma$ is a torsion-free elementary amenable group which acts nilpotently on $H^{*}(\tilde{X})$, then Lee and Park [8] showed that $\chi(X)=0$. If $X$ is aspherical, then any subgroup of $\Gamma$ acts nilpotently on $H^{*}(\tilde{X})$. Hence Theorem 1.1 generalizes the results of Cheeger and Gromov [2] and Eckmann [5]. As elementary amenable groups are amenable, Theorem 1.1 also generalizes the result of Lee and Park [8]. If $\Gamma$ has finite virtual cohomological dimension and contains a nontrivial torsion-free elementary amenable normal subgroup which acts nilpotently on $H^{*}(\tilde{X})$, then $\chi(X)=0$. In fact, Hillman and Linnell [6] showed that any nontrivial torsion-free elementary amenable group of finite virtual cohomological dimension contains a nontrivial Abelian characteristic subgroup. Applying Eckmann's result [4] yields $\chi(X)=0$. Note that it is not known whether $X=\tilde{X} / \Gamma$ being compact implies that $\Gamma$ has finite virtual cohomological dimension.

The proof of Theorem 1.1 is based on results concerning the von Neumann dimension of simplicial $\ell_{2}$-cohomolgy spaces. Theorem 1.2 is another type of vanishing result for the Euler characteristic $\chi(X)$ of $X$.

\section{Simplicial $\ell_{2}$-cohomolgy}

Let $G$ be a countable group and let $\ell_{2}(G)$ denote the Hilbert space of real valued square summable functions on $G$. A pre-Hilbert space $P$ is called a Hilbert $G$-module if:

(i) $G$ acts on $P$ by isometries, and

(ii) $P$ is $G$-equivariantly isometric to a subspace of the tensor product $\ell_{2}(G) \otimes H$ of the Hilbert space $\ell_{2}(G)$ and some Hilbert space $H$ with trivial $G$-action.

To such a $P$, following von Neumann and Atiyah (see [1] and [3]), one can attach a nonnegative extended real number, $0 \leq \operatorname{dim}_{G} P \leq \infty$, called the von Neumann dimension of $P$, which is independent of the particular identification with a subspace of $\ell_{2}(G) \otimes H$ (See Remark 2.3). If $P \neq 0$, then $\operatorname{dim}_{G} P>0$. Moreover, the von Neumann dimension of a pre-Hilbert space is equal to that of its completion. As usual,

$$
\operatorname{dim}_{G}\left(P_{1} \oplus P_{2}\right)=\operatorname{dim}_{G} P_{1}+\operatorname{dim}_{G} P_{2}
$$

For further background on Hilbert $G$-modules we refer the reader to $[1,2,3]$.

Let $G$ be a countable group and $Y$ a connected simplicial complex on which $G$ acts freely and simplicially. Denote by $Y_{(n)}$ the set of all $n$-simplices of $Y$. Define $C_{(2)}^{n}(Y)=\left\{c \in C^{n}(Y, \mathbb{R}) \mid \sum_{s \in Y_{(n)}} c(s)^{2}<\infty\right\}$ and call it the space of $\ell_{2}$-cochains. Then $C_{(2)}^{n}(Y) \cong \ell_{2}(G) \otimes H_{n}$ where $H_{n}$ is a Hilbert space having a set $S_{n}$ of representatives of $Y_{(n)} \bmod G$ as a basis. Hence $C_{(2)}^{n}(Y)$ is a free Hilbert $G$-module and $\operatorname{dim}_{G} C_{(2)}^{n}(Y)=$ cardinality $\left|S_{n}\right|$ of $S_{n}$. It is clear that the differentials $\delta^{n}: C_{(2)}^{n}(Y) \rightarrow C_{(2)}^{n+1}(Y)$ commute with the $G$-action. We define the simplicial $\ell_{2}$-cohomolgy spaces by

$$
H_{(2)}^{n}(Y: G)=\operatorname{Ker} \delta^{n} / \operatorname{Im} \delta^{n-1}
$$


and we define the (reduced) simplicial $\ell_{2}$-cohomology spaces by

$$
\bar{H}_{(2)}^{n}(Y: G)=\operatorname{Ker} \delta^{n} / \overline{\operatorname{Im} \delta^{n-1}} .
$$

Note that $C_{(2)}^{n}(Y) \supset \operatorname{Ker} \delta^{n} \cong \overline{\operatorname{Im} \delta_{n-1}} \oplus \bar{H}_{(2)}^{n}(Y: G)$, and hence $\operatorname{Ker} \delta^{n}$, $\operatorname{Im} \delta^{n-1}$, and $\operatorname{Im} \delta^{n-1}$ are Hilbert $G$-modules. In particular $\bar{H}_{(2)}^{n}(Y: G)$ acquires the structure of a Hilbert $G$-module and hence its von Neumann dimension is defined, denoted by $h^{n}(Y: G)$, and called the $n t h \ell_{2}$-Betti number. Moreover there is a natural $G$-equivariant map [2]

$$
\rho: \bar{H}_{(2)}^{*}(Y: G) \rightarrow H^{*}(Y, \mathbb{R})
$$

Remark 2.1. If $Y$ is a connected simplicial complex on which $G$ acts freely and simplicially so that the quotient $Y / G$ is compact, then

$$
\begin{aligned}
\chi(Y / G) & =\sum(-1)^{n}\left|S_{n}\right|=\sum(-1)^{n} \operatorname{dim}_{G} C_{(2)}^{n}(Y) \\
& =\sum(-1)^{n} \operatorname{dim}_{G} \bar{H}^{n}(Y: G)=\sum(-1)^{n} h^{n}(Y: G) .
\end{aligned}
$$

The first equality follows from the fact that $Y / G$ is a finite complex and the third equality follows from the fact that the cochain complex $\left\{C_{(2)}^{*}(Y)\right\}$ of Hilbert $G$-modules is finite.

Proposition 2.2. For an infinite subgroup $A$ of $G$, any Hilbert $G$-module with trivial A-action is the zero module.

Proof. Let $P$ be a Hilbert $G$-module with trivial $A$-action and a $G$-equivariant embedding $P \hookrightarrow \ell_{2}(G) \otimes H$. We may assume that $P$ is a Hilbert space. Let $\left\{h_{i}\right\}$ be a Hilbert basis of $H$ and let $p_{i}: \ell_{2}(G) \otimes H \rightarrow \ell_{2}(G)$ be the projection $1 \otimes r h_{i} \mapsto r \cdot 1$. With $P_{0}=P$, we define inductively $P_{i+1}$ and $I_{i+1}$ to be the kernel and the closure of the image, respectively, of $p_{i+1} \circ j_{i}: P_{i} \hookrightarrow \ell_{2}(G) \otimes H \rightarrow \ell_{2}(G)$. I.e., $P_{i}=\operatorname{ker} p_{1} \cap \ldots \cap \operatorname{ker} p_{i} \cap P$ and then $I_{i+1}$ is the closure of the image of $P_{i}$ in $\ell_{2}(G)$. Then $P=\sum I_{i}$ and $I_{i}$ is a Hilbert $G$ module with a $G$-equivariant embedding $I_{i} \hookrightarrow \ell_{2}(G)([3])$. Since $p_{i+1} \circ j_{i}$ is $G$ - and so $A$-equivariant, the $A$-action on $I_{i}$ is trivial.

Note that $\ell_{2}(G)=\ell_{2}(A) \otimes \mathcal{H}$ where $\mathcal{H}$ is the Hilbert space having $G / A$ as its Hilbert basis. By the same argument as above each Hilbert $G$-module $I_{i}$ has a decomposition $I_{i}=\sum J_{i,}$ by Hilbert $A$-modules such that $J_{i, j} \subset \ell_{2}(A)$ with trivial $A$-action. Now it suffices to show that each $J_{i_{j}}=0$.

Let $J \subset \ell_{2}(A)$ with trivial $A$-action. Every element of $J$ is of the form $\sum_{x \in A} a_{x} x$ where $\sum_{x \in A}\left|a_{x}\right|^{2}<\infty$. If $a_{x} \neq 0$ for some $x \in A$, then because of the trivial action by $A$ $a_{1}=a_{x} \neq 0$. For any $y \in A, a_{y}=a_{1} \neq 0$. Hence $\sum_{x \in A} a_{x} x=\sum_{x \in A} a_{1} x$, so $\sum_{x \in A}\left|a_{x}\right|^{2}=$ $\sum_{x \in A}\left|a_{1}\right|^{2}=\infty$. This implies $\sum_{x \in A} a_{x} x=0$. Hence $J=0$. 
Remark 2.3. As in the proof of Proposition 2.2, any Hilbert $G$-module $P$ which is a Hilbert space is isomorphic to $\sum I_{i}$ where $I_{i} \hookrightarrow \ell_{2}(G)$. Write $1=e_{i}+\left(1-e_{i}\right)$ where $e_{i} \in I_{i}$ and $1-e_{i} \in I_{i}^{\perp}$. Then $e_{i}=\sum_{x \in G}\left\langle e_{i}, x\right\rangle x$ where $\langle$,$\rangle is the inner product on \ell_{2}(G)$. The trace of $e_{i},\left\langle e_{i}, 1_{G}\right\rangle$, i.e., the coefficient of the identity $1_{G}$ of $G$, is the von Neumann dimension of $I_{i}$. The von Neumann dimension of $P$ is then $\operatorname{dim}_{G} P=\sum \operatorname{dim}_{G} I_{i}$.

\section{Nilpotent modules}

Definition 3.1. Let $A$ be a subgroup of $G$ and let $M$ be a $\mathbb{Z} G$-module. Then we say that $A$ acts nilpotently on $M$ if there exists a finite filtration $0=M^{(0)} \subset M^{(1)} \subset \ldots$ $\subset M^{(k-1)} \subset M^{(k)}=M$ by $\mathbb{Z} A$-modules such that $A$ acts trivially on the associated graded module Gr $M=\left\{M^{(i)} / M^{(i-1)} \mid i=1, \ldots, k\right\}$.

Remark 3.2. The $M^{(i)}$ in Definition 3.1 can be chosen such that $M^{(i)} / M^{(i-1)}$ consists of all elements of $M / M^{(i-1)}$ fixed under the action of $A$.

Proposition 3.3 [4, Proposition 1.1]. Let $M$ be a $\mathbb{Z G - m o d u l e . ~ S u p p o s e ~ a ~ s u b g r o u p ~} A$ of $G$ acts nilpotently on $M$ so that a filtration $\left\{M^{(i)} \mid i=0, \ldots, k\right\}$ of $M$ is chosen as in Remark 3.2. If $A$ is a normal subgroup of $G$, then the $M^{(i)}$ are $\mathbb{Z} G$-submodules of $M$.

Proof. This is trivial for $i=0$, and we assume that it holds for $i-1$ $(i=1,2, \ldots, k)$. For any $h \in M^{(i)}, a \in A$, and $x \in G$, as $A$ is normal in $G$ we have $x^{-1} a x \in A$, and as $A$ acts trivially on $M^{(i)} / M^{(i-1)}$ we have $a x h=x\left(x^{-1} a x\right) h=x\left(h+h^{\prime}\right)$ with $h^{\prime} \in M^{(i-1)}$. Since $x h^{\prime} \in M^{(i-1)}$, axh $=x h+h^{\prime \prime}$ with $h^{\prime \prime} \in M^{(i-1)}$. Thus $a \in A$ fixes the element $x h+M^{(i-1)}$ in $M / M^{(i-1)}$, and hence $x h \in M^{(i)}$.

Theorem 3.4. Let $G$ be a countable group and let $Y$ be a connected simplicial complex on which $G$ acts freely and simplicially so that the quotient $Y / G$ is compact. If $G$ contains an infinite normal subgroup $A$ which acts nilpotently on $H^{*}(Y)$, then the natural $G$ equivariant map $\rho: \bar{H}_{(2)}^{*}(Y: G) \rightarrow H^{*}(Y, \mathbb{R})$ is trivial.

Proof. Let $K$ be the kernel of $\rho$, and let $\bar{M}=\bar{H}_{(2)}^{*}(Y: G)$ and $M=H^{*}(Y, \mathbb{R})$. Take a filtration $\left\{M^{(i)}\right\}_{i=0}^{k}$ of $M$ given by the nilpotent action of $A$ on $M$ as in Remark 3.2. By Proposition 3.3, the $M^{(i)}$ are $\mathbb{R} G$-modules. Let $\bar{M}^{(i)}=\rho^{-1}\left(M^{(i)}\right)$ for $i=0,1, \ldots, k$. Then we have exact sequences $0 \rightarrow K \rightarrow \bar{M}^{(i)} \rightarrow M^{(i)}$, and $\bar{M}^{(i)} / \bar{M}^{(i-1)} \cong\left(\bar{M}^{(i)} / K\right) /\left(\bar{M}^{(i-1)} / K\right) \hookrightarrow$ $M^{(i)} / M^{(i-1)}$ so $A$ acts trivially on $\bar{M}^{(i)} / \bar{M}^{(i-1)}$; by assuming that each $\bar{M}^{(i)}$ is a Hilbert space, if it is necessary, we obtain a decomposition of $\bar{M}$ by $\ell_{2}(G)$-modules:

$$
\bar{M}=\bar{M}^{(k)} \oplus\left[\bar{M}^{(k)}\right]^{\perp}=\cdots=K \oplus K^{\perp} \oplus\left[\bar{M}^{(1)}\right]^{\perp} \oplus \cdots \oplus\left[\bar{M}^{(k)}\right]^{\perp},
$$

where $A$ acts trivially on the factors $K^{\perp},\left[\bar{M}^{(1)}\right]^{\perp}, \cdots$, and $\left[\bar{M}^{(k)}\right]^{\perp}$. By Proposition 2.2, $\bar{M}=K$. Hence $\rho$ is a trivial map. 
Proof of Theorem 1.1. Let $Y=\tilde{X}$ and $G=\pi_{1}(X)$. Since $G$ is an infinite amenable group and $Y / G$ is a finite complex, by Lemma 3.1 of [2] the natural $G$-equivariant map $\rho: \bar{H}_{(2)}^{*}(Y: G) \rightarrow H^{*}(Y, \mathbb{R})$ is injective. On the other hand, by Theorem 3.4, $\rho$ is the trivial map. this implies $\bar{H}_{(2)}^{*}(Y: G)=0$ and in particular $\chi(X)=0$.

Corollary 3.5. If $\Gamma=\pi_{1}(X)$ is an infinite amenable group and if $\tilde{X}$ is homotopic to an even dimensional sphere $S^{2 k}$, then $\chi(X)=0$. If, in addition, $\Gamma$ has finite virtual cohomological dimension $\operatorname{vcd}(\Gamma), \infty$, then the rational Euler characteristic $\chi(\Gamma)$ of $\Gamma$ vanishes.

Proof. Since $H^{2 k}(\tilde{X})=\mathbb{Z}$, the kernel $\Gamma^{\prime}$ of the induced action homomorphism $\Gamma \rightarrow \operatorname{Aut}\left(H_{2 k}(\tilde{X})\right)=\operatorname{Aut}(\mathbb{Z}) \cong \mathbb{Z}_{2}$ has index at most 2 in $\Gamma$ and acts trivially, and hence nilpotently, on $H^{*}(\tilde{X})$. By Theorem $1.1 \chi\left(\tilde{X} / \Gamma^{\prime}\right)=0$. Thus $\chi(X)=0$. If $\operatorname{vcd}(\Gamma)<\infty$, then $\chi(\Gamma)$ is defined and $\chi(X)=\chi(\Gamma) \cdot \chi(\tilde{X})$ (See $[7,8])$. Hence $\chi(\Gamma)=0$.

\section{Proof of Theorem 1.2}

Let $\Pi$ be a group and let $G$ be a $\Pi$-group, i.e., a group with $\psi$-action $\psi: \Pi \rightarrow \operatorname{Aut}(G)$. If $G$ is a normal subgroup of $\Pi$ we take $\psi(x) g=x \cdot g \cdot x^{-1}$. By $\Pi_{2} G$ we mean the normal $\Pi$ subgroup of $G$ generated by all elements of the form $(\psi(x) g) \cdot g^{-1}$, where $x \in \Pi$ and $g \in G$. Inductively we define $\Pi_{n} G=\Pi_{2}\left(\Pi_{n-1} G\right)$. The $\Pi$-group $G$ is called $\Pi$-nilpotent if $\Pi_{n} G=0$ for some $n$. A nilpotent group $G$ is a $G$-nilpotent group.

Let $\tilde{X}_{A}$ denote the covering space of $X$ corresponding to the normal subgroup $A$ of $\Gamma=\pi_{1}(X)$. Then $\Gamma / A$ acts on $\tilde{X}_{A}$ freely and simplicially with quotient $X$, and hence $\Gamma$ acts on $\tilde{X}_{A}$ by composition with the quotient map $\Gamma \rightarrow \Gamma / A$. Consider the cohomology spectral sequence corresponding to the fibration $\tilde{X} \rightarrow \tilde{X}_{A} \rightarrow K(A, 1)$;

$$
E_{2}^{p, q}=H^{p}\left(A ; H^{q}(\tilde{X})\right) \Rightarrow H^{p+q}\left(\tilde{X}_{A}\right) .
$$

We will first show that $\Gamma$ acts nilpotently on $E_{2}^{p, q}=H^{p}\left(A ; H^{q}(\tilde{X})\right)$ and hence on $H^{*}\left(\tilde{X}_{A}\right)$.

Given an element $\alpha \in \Gamma$, let $h: \tilde{X}_{A} \rightarrow \tilde{X}_{A}$ be the associated deck transformation. This $h$ is not necessarily base point preserving, but it can be homotoped to a map $h^{\prime}$ which preserves base point so that $h_{*}^{\prime}: \pi_{1}\left(\tilde{X}_{A}\right) \rightarrow \pi_{1}\left(\tilde{X}_{A}\right)$ is conjugation by $\alpha$. Then $h^{\prime}$ can be lifted to a map $h^{\prime \prime}: \tilde{X} \rightarrow \tilde{X}$ which preserves base point and is freely homotopic to the deck transformation of $\tilde{X}$ associated with $\alpha$. Also there is an associated based map $h^{\prime}: K(A, 1) \rightarrow K(A, 1)$ so that $h_{*}^{\prime}: \pi_{1}(K(A, 1))=A \rightarrow \pi_{1}(K(A, 1))=A$ is conjugation by $\alpha$. This is how $\Gamma$ acts on the fibration

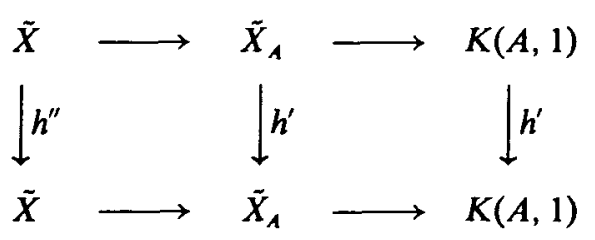


each square commuting up to based homotopy, in such a way that the induced actions by $\Gamma$ on $H^{*}(\tilde{X})$ and $H^{*}\left(\tilde{X}_{A}\right)$ are the natural actions. Hence $\Gamma$ acts on the $E_{2}$ term of the spectral sequence corresponding to the fibration $\tilde{X} \rightarrow \tilde{X}_{A} \rightarrow K(A, 1)$ and the boundary maps $d_{r}$ are $\Gamma$-module maps. Since $A$ is $\Gamma$-nilpotent, $\Gamma$ acts nilpotently on $H^{p}(A ; T)$ for any trivial $A$-module $T$. Since $\Gamma$ acts nilpotently on $H^{q}(\tilde{X})$, we take a finite filtration $0=M^{(0)} \subset M^{(1)} \subset \cdots \subset M^{(k)}=H^{q}(\tilde{X})$ by $\Gamma$-submodules so that $\Gamma$ acts trivially on $\left\{M^{(i)} / M^{(i-1)}\right\}_{i=1}^{k}$. In the long cohomology exact sequence of $A$ associated with the exact sequence of coefficient modules $0 \rightarrow M^{(1)} \rightarrow M^{(2)} \rightarrow M^{(2)} / M^{(1)} \rightarrow 0, \Gamma$ acts nilpotently on $H^{p}\left(A ; M^{(1)}\right)$ and $H^{p}\left(A ; M^{(2)} / M^{(1)}\right)$. Hence $\Gamma$ acts nilpotently on $H^{p}\left(A ; M^{(2)}\right)$. By induction, $\Gamma$ acts nilpotently on $E_{2}^{p, q}=H^{p}\left(A ; H^{q}(\tilde{X})\right)$, and hence on the abutment $H^{p+q}\left(\tilde{X}_{A}\right)$ of the sequence.

In all, we have shown that the infinite amenable group $\Gamma / A$ acts freely and simplicially on $\tilde{X}_{A}$ with compact quotient $X$ and acts nilpotently on $H^{*}\left(\tilde{X}_{A}\right)$. By Theorem 3.4 , the reduced $\ell_{2}$-cohomology spaces $\bar{H}_{(2)}^{*}\left(\tilde{X}_{A} ; \Gamma / A\right)$ are trivial and hence $\chi(X)=\chi\left(\tilde{X}_{A} /(\Gamma / A)\right)=0$.

Acknowledgement. The author would like to thank the referee for making careful corrections of a few expressions in his original version.

\section{REFERENCES}

1. M. F. Aтiуaн, Elliptic operators, discrete groups, and von Neumann algebras, Asterisque 32-33 (1976), 43-72.

2. J. Cheeger and M. Gromov, $L_{2}$-cohomology and group cohomology, Topology 25 (1986), 189-215.

3. J. M. Cohen, Von Neumann dimension and the homology of covering spaces, Quart. J. Math. Oxford Ser. (2) 30 (1979), 133-142.

4. B. ECKMANN, Nilpotent group action and Euler characteristic (Lecture Note in Mathematics, 1248, 1985), 120-123.

5. B. Eckmann, Amenable groups and Euler characteristic, Comment. Math. Helv. 67 (1992), 383-393.

6. J. A. Hillman and P. A. Linnell, Elementary amenable groups of finite Hirsch length are locally-finite by virtually solvable, J. Austral. Math. Soc. Ser. A 52 (1992), 237-241.

7. J. B. LeE, Transformation groups on $S^{n} \times \mathbb{R}^{m}$, Topology Appl. 53 (1993), 187-204.

8. J. B. LeE and C.-Y. PARK, Nilpotent action by an elementary amenable group and Euler characteristic, Bull. Korean Math. Soc. 33 (1996), 253-258.

9. A. L. Paterson, Amenability (Mathematical Surveys and Monographs, 29, Amer. Math. Soc., Providence, R. I., 1994).

Department of Mathematics

SOGANG UNIVERSITY

SEOUL $121-742$

KOREA

E-mail address: jlee@ccs.sogang.ac.kr 